\title{
Influence of magnet size on magnetically engineered field-induced superconductivity
}

\author{
W. Gillijns, ${ }^{1, *}$ M. V. Milošević, ${ }^{2,3}$ A. V. Silhanek, ${ }^{1}$ V. V. Moshchalkov, ${ }^{1, \dagger}$ and F. M. Peeters ${ }^{3, \sharp}$ \\ ${ }^{1}$ INPAC-Institute for Nanoscale Physics and Chemistry, Nanoscale Superconductivity and Magnetism and Pulsed Fields Group, \\ K.U. Leuven, Celestijnenlaan 200D, B-3001 Leuven, Belgium \\ ${ }^{2}$ Department of Physics, University of Bath, Claverton Down, Bath BA2 7AY, United Kingdom \\ ${ }^{3}$ Departement Fysica, Universiteit Antwerpen, Groenenborgerlaan 171, B-2020 Antwerpen, Belgium
}

(Received 27 July 2007; published 20 November 2007)

\begin{abstract}
We investigate experimentally and theoretically the superconducting properties of an $\mathrm{Al}$ thin film covering a periodic array of $\mathrm{Co} / \mathrm{Pt}$ magnetic disks with out-of-plane magnetization for different radii of the magnetic disks and constant period of the magnetic lattice. The presence of the arrays of magnetic dots leads to a quantized displacement of the normal/superconducting phase boundary along the magnetic field axis, with each step corresponding to a flux-quantum per unit cell of the magnetic lattice. We demonstrate that this so-called field-induced superconductivity is strongly dependent not only on the chosen magnetic material and its magnetization $M$ but also on the radius $R$ of the constructed magnetic disks. Since field-induced superconductivity is directly linked to the nucleation of vortex-antivortex $(\mathrm{V}-\mathrm{AV})$ pairs, a theoretical $M-R$ equilibrium phase boundary is presented, delimiting regions of different numbers of induced V-AV pairs per magnet. A good qualitative and quantitative agreement is found between theory and experiment.
\end{abstract}

DOI: 10.1103/PhysRevB.76.184516

PACS number(s): 74.78.Fk, 74.25.Dw

\section{INTRODUCTION}

It is a well established fact that the properties of a superconductor (S) can be modified by the interaction with a nearby ferromagnet $(\mathrm{F})$, either through the electron exchange when in direct contact (i.e., proximity effect $)^{1}$ or by the presence of the stray fields emanating from the ferromagnet. ${ }^{2} \mathrm{By}$ electrically insulating the ferromagnet from the superconductor, the latter mechanism dominates and the electromagnetic interaction determines the properties of the hybrid system. As a result, the typical linear decrease of the critical temperature $T_{c}$ with increasing uniform magnetic field $H$ for a single $\mathrm{S}$ layer can be strongly modified in S/F heterostructures. In these hybrid systems, the superconducting order parameter is spatially modulated, leading to regions of localized superconductivity where the effective magnetic field is minimal, ${ }^{3,4}$ i.e., where the stray fields of the ferromagnets and the external magnetic field compensate each other. These compensation effects give rise to exotic $T_{c}(H)$ phase boundaries in S/F bilayers, as recently reported in Refs. 5 and 6. However, in the case of particularly large local magnetic stray fields, screening effects become insufficient and vortices are generated in the superconductor in order to lower the total energy of the system. ${ }^{7,8}$ Due to the properties of the magnetic field of a ferromagnet and flux conservation, an isolated vortex cannot exist; each vortex is accompanied by an antivortex, and both nucleate together as a vortexantivortex (V-AV) pair. $^{7}$

In the case that a superconductor is covered with an array of magnetic dots with out-of-plane magnetization, regular vortex-antivortex lattices are created. ${ }^{9-13}$ It was found that the resulting inhomogeneous field profile leads to a more robust superconducting condensate at nonzero fields, leading to field-induced superconductivity. Indeed, by applying an external magnetic field, either the antivortices, located interstitially between the magnets, or the vortices on top of the dots can be compensated. ${ }^{14}$ As a consequence, regions with small local effective field will appear for nonzero external fields at the expense of having high effective field in other regions. This effect manifests itself as a maximal critical temperature shifted away from $H=0$. It has recently been demonstrated that the number of nucleated V-AV pairs and accordingly the shift of the phase boundary can be tuned by controlling the magnetization of the dots. ${ }^{12,13,15}$ Unfortunately, the maximal shift of the phase boundary is limited by the saturation magnetization of the magnetic dots. A way to overcome this limitation is to increase the magnetic flux from each magnet by increasing the size of the dots. However, by doing so, less space becomes available for the nucleation of antivortices between the dots, possibly conspiring against their stabilization in the superconducting condensate.

In this paper, we investigate the combinatorial effect of changing both the magnetization $M$ and the radius $R$ of the magnetic dots on the superconducting properties of an $\mathrm{Al}$ film deposited on top of a periodic array of such dots. In particular, we study the normal/superconducting phase boundary experimentally, which is also supported by a theoretical study of the creation of V-AV pairs. The agreement between simulations and experiment supports the interpretation that field-induced superconductivity is mainly a consequence of the spontaneous V-AV generation as a result of the magnetic array.

\section{SAMPLE CHARACTERIZATION}

The samples consist of Al films covering a square array of magnetic dots of radius $R$ spanning from 300 to $680 \mathrm{~nm}$. The lattice parameter $W=2 \mu \mathrm{m}$ of the array is kept constant for all samples (see the inset of Fig. 1). Standard electron beam lithography is used to laterally define the dots in a resist mask. A multilayer of Co and $\mathrm{Pt}$ is subsequently evaporated by molecular beam epitaxy (MBE) with the following structure: $\mathrm{Pt}(2.5 \mathrm{~nm})+[\mathrm{Co}(0.4 \mathrm{~nm}) / \mathrm{Pt}(1.0 \mathrm{~nm})]_{10}$. This multilayered composite exhibits out-of-plane magnetization. ${ }^{16} \mathrm{Af}-$ 


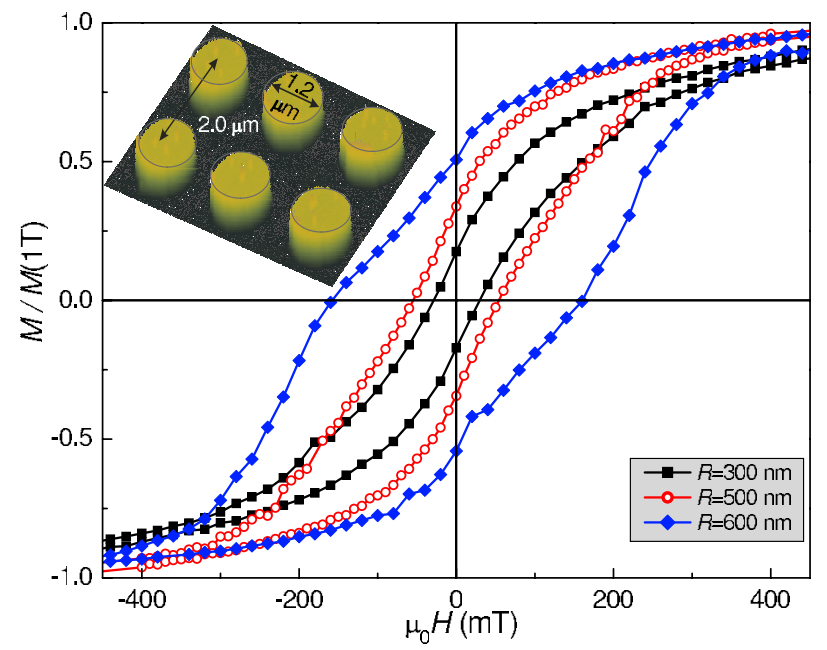

FIG. 1. (Color online) Magnetization loops $M(H)$ of arrays of $\mathrm{Co} / \mathrm{Pt}$ dots with different radii. The inset shows an atomic force microscopy image of a typical dot array.

ter lift-off, the array of $\mathrm{Co} / \mathrm{Pt}$ dots is covered by a $\mathrm{Si}$ film of $5 \mathrm{~nm}$ to suppress proximity effect, followed by an Al film with a thickness of $50 \mathrm{~nm}$. Both the $\mathrm{Si}$ and the $\mathrm{Al}$ are evaporated in a MBE system and patterned in a transport bridge.

The magnetic properties of the dots are characterized by measuring the out-of-plane magnetization loops of the dots in a superconducting quantum interference device magnetometer. The hysteresis loops for different dot sizes are displayed in Fig. 1 after normalization by the magnetization $M$ at $1.0 \mathrm{~T}$. The deviation from the square shaped hysteresis loops for the reference plain film of $\mathrm{Co} / \mathrm{Pt}$ (not shown) is a result of the patterning of the $\mathrm{Co} / \mathrm{Pt}$ into the dots.

Starting from a demagnetized state, the magnetization of the dots can be increased in arbitrary steps by applying a certain excursion field and returning back to zero field (i.e., by constructing minor hysteresis loops). In this way, the magnetization $M$ can be controlled from $M \approx 0$ (demagnetized) to $M=M_{\text {rem }}$ by choosing the correct excursion field. This procedure, described in detail in Ref. 15, allows us to tune the magnetization and hence the electromagnetic influence of the dots on the $\mathrm{Al}$ films. The typical excursion field needed to change the magnetization of the $\mathrm{Co} / \mathrm{Pt}$ dots is higher than the upper critical field of the superconductor, and we can assume a constant magnetization during measurements of the superconducting state.

We investigate the influence of the magnetic dots on the superconducting layer by means of transport measurements. From the obtained normal/superconducting phase boundaries of different samples, we determined the critical temperature $T_{c}$ of approximately $1.35 \mathrm{~K}$ and a coherence length $\xi(0)$ $\sim 115 \mathrm{~nm}$.

\section{RESULTS AND DISCUSSION}

Let us first briefly review the effects of changing the magnetization $M$ of the dots on the superconducting state. ${ }^{15}$ The evolution of the magnetoresistance $\rho(H)$ for a sample with an



FIG. 2. Normalized magnetoresistance $\rho(H) / \rho_{n}$, with $\rho_{n}$ the normal state resistance, of $R=400 \mathrm{~nm}$ sample for increasing magnetization values and temperatures close to $T_{c}$. The curves are shifted by 0.5 for clarity.

array of dots with radius $R=400 \mathrm{~nm}$ as $M$ is progressively increased is summarized in Fig. 2. These $\rho(H)$ curves are normalized by the normal state resistance and are shifted vertically for clarity. It can be seen that for small magnetization values $\left(M<1.69 \times 10^{5} \mathrm{~A} / \mathrm{m}\right)$ the lowest resistance [and accordingly the highest critical temperature $T_{c}^{\max }$ $\left.=\max _{H}\left\{T_{c}(H)\right\}\right]$ is obtained at zero field. As $M$ increases, an incipient local minimum develops at the first matching field $H_{1}$, where $H_{n}=n \frac{\phi_{0}}{a^{2}}$ corresponds to a magnetic field at which on average $n$ flux quanta $\phi_{0}$ occupy one unit cell. The resulting asymmetric phase boundary is a consequence of the induced screening currents in the superconducting film due to the magnetized dots. These supercurrents give rise to a field polarity dependent pinning landscape. Strong pinning is found if the magnetization of the dot and the polarity of the vortex are parallel (vortices are attracted by the magnets), whereas weak pinning occurs for an antiparallel alignment (vortices are repelled and caged at interstitial sites). ${ }^{17,18}$ For a magnetization of approximately $M=2.42 \times 10^{5} \mathrm{~A} / \mathrm{m}, T_{c}$ is practically the same at $H_{0}$ and $H_{1}$ (see Fig. 2). The magnetization at which this crossover of $T_{c}^{\max }$ occurs is defined as the critical magnetization necessary to shift the phase boundary by one matching field. For larger magnetic moments, the maximum critical temperature $T_{c}^{\max }$ is uniquely defined at $H_{1}$. Essentially, this means that superconductivity in the studied hybrid structure has been enhanced in the presence of an 




FIG. 3. (Color online) Experimental $M-R$ phase boundary for an array of magnetic dots with periodicity of $2 \mu \mathrm{m}$ delimiting the regions for which $T_{c}^{\max }$ is displaced by no, one, and two matching fields. For small magnetization and small radius, no shift is present. By increasing the radius and/or the magnetization, the superconducting phase boundary moves toward higher field values. Solid lines represent the theoretical prediction (see below).

applied magnetic field, contrary to the conventional behavior.

The main mechanism behind this field-induced superconductivity is the compensation between the uniform applied field and the inhomogeneous stray field generated by the magnetic array. For positively magnetized dots, a positive $z$ component of the magnetic field will be present above the dots, while a negative component is located in between the dots. By applying a positive external magnetic field, the field in between the dots will be compensated, hence leading to a maximum $T_{c}$ at nonzero field. By increasing the magnetization, the optimal compensation shifts to higher applied fields, and we found a maximal field shift of $2 \mathrm{H}_{1}$ for this dot size. This maximum is basically determined by the material dependent saturation magnetization of the dots and the geometrical details of the $\mathrm{S} / \mathrm{F}$ hybrid. It is precisely the influence of the geometrical parameters that we investigate in this paper. We restrict ourselves to changing only the radius of the magnetic dots while keeping their thickness and shape unchanged.

We investigate the applied field values at which $T_{c}^{\max }$ is reached as a function of the magnetization $M$ for five different radii of the dots, namely, $R=300,400,500,600$, and $680 \mathrm{~nm}$. For each size of the dots, we measured the magnetoresistance while sweeping up their magnetization (similar to the data shown in Fig. 2). In this way, for each dot size $R$, we determined the critical magnetization needed to displace $T_{c}^{\max }$ from $H_{n}$ to $H_{n+1}$. Gathering all data through this procedure enables us to build a complete $M-R$ phase boundary, as shown in Fig. 3. Here, the symbols indicate the magnetization at which equal values of $T_{c}^{\max }$ are found at two consecutive matching fields. The regions in between correspond to parameters for which the field position of $T_{c}^{\max }$ is fixed at a particular matching field. The theoretically calculated solid lines represent the parameters at which an extra $\mathrm{V}$-AV pair is generated (see below). For the smallest dots, a maximal shift of $H_{1}$ can be reached before reaching saturation, whereas $T_{c}^{\max }$ can be displaced up to $H_{6}=6 H_{1}$ for the largest dots. It is important to note that the temperature at which the magnetoresistance is measured is lowered as the magnetization is increased, since the absolute value of $T_{c}^{\max }$ decreases while the magnetization is increased. This follows from the fact that inhomogeneous stray field of the dots can never be perfectly compensated by the applied field, and remaining screening currents in the sample roughly scale with the magnetization of the dots. ${ }^{19}$ As a result, there is an intrinsic limit at which $T_{c}^{\max }=0$, and hence no further shift in the critical field can be achieved.

We complement our experimental study with a theoretical approach in the framework of the nonlinear GinzburgLandau (GL) theory. The dots are modeled as disks magnetized in the positive $z$ direction lying on top of a thin film superconductor ( $x y$ plane), which is equivalent to having the superconductor on top of the dots as in the experiment. It is assumed that the magnetic disks are made of a hard magnet whose uniform magnetic moment is not affected by nearby circulating supercurrents. Additionally, we assume a thin insulating layer in between the dots and the superconducting film.

If the superconductor thickness $d$ is smaller than the coherence length $\xi$ and the penetration depth $\lambda$, the GL equations may be averaged over the film thickness:

$$
\begin{gathered}
\left(-i \vec{\nabla}_{2 \mathrm{D}}-\vec{A}\right)^{2} \Psi=\Psi\left(1-|\Psi|^{2}\right), \\
-\Delta_{3 \mathrm{D}} \vec{A}=\frac{d}{\kappa^{2}} \delta(z) \vec{j}_{2 \mathrm{D}},
\end{gathered}
$$

where

$$
\vec{j}_{2 \mathrm{D}}=\frac{1}{2 i}\left(\Psi^{*} \vec{\nabla}_{2 \mathrm{D}} \Psi-\Psi \vec{\nabla}_{2 \mathrm{D}} \Psi^{*}\right)-|\Psi|^{2} \vec{A}
$$

is the density of superconducting currents, $\Psi$ is the superconducting order parameter, $\kappa=\lambda / \xi$ is the GL parameter, $\delta(x)$ is the Dirac delta function, and $\vec{A}$ is the total vector potential resulting from both the magnetic dots and the induced supercurrents. In the above equations, the distance is measured in units of the coherence length, the vector potential in $c \hbar / 2 e \xi$, and the magnetic field in $H_{c 2}=c \hbar / 2 e \xi^{2}$ $=\kappa \sqrt{2} H_{c}$, where $H_{c}$ is the thermodynamic critical field. The indices $2 \mathrm{D}$ and $3 \mathrm{D}$ refer to two- and three-dimensional operators, respectively. Equations (1)-(3) are accompanied by standard periodic boundary conditions. ${ }^{20}$

The $M-R$ phase boundary is then calculated by looking for stable vortex-antivortex states for each size of the dots and their magnetization. To find these different vortex configurations including the metastable states, we search for the steady-state solutions of Eqs. (1) and (2) starting from different randomly generated initial configurations. Then, we slowly increase or decrease the magnetization of the magnetic disks $M$ and recalculate each time the exact vortex structure. We do this for each vortex configuration in a magnetization range where the number of vortices remains constant and repeat the whole process when the size of the magnets is changed. By comparing the Gibbs free energies of the 


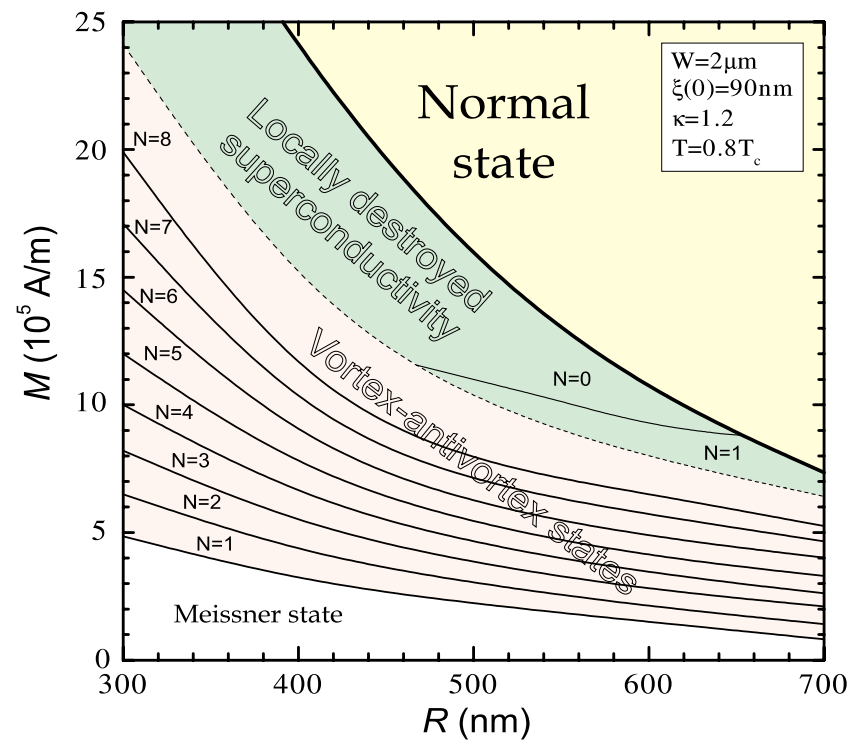

FIG. 4. (Color online) Ground-state vortex-antivortex phase diagram at a temperature of $T=0.8 T_{c}$, as a function of the radius of the magnetic dots and their magnetization, for a fixed period of the magnetic lattice $W=2 \mu \mathrm{m}$. $N$ denotes the number of induced V-AV pairs per dot.

different vortex configurations, we obtain the ground state $\left(\mathcal{G}=V^{-1} \int_{V}\left[2\left(\vec{A}-\vec{A}_{0}\right) \cdot \vec{j}-|\Psi|^{4}\right] d V\right.$, where $V$ is the volume of the simulation region and $A_{0}$ stands for the vector potential of the magnetic dots only).

Our theoretical calculations, in general, show that the crossing of $T_{c}^{\max }$ from $H_{n}$ to $H_{n+1}$ is always linked to the number of magnet-induced $\mathrm{V}-\mathrm{AV}$ pairs. Intuitively, $T_{c}^{\max }$ is reached when the number of externally added flux quanta $n$ per unit cell (thus, $H=H_{n}$ ) matches the number of antivortices induced by the magnet with given magnetization $M$. In that case, optimal compensation in between the dots is reached, since added vortices annihilate with the present antivortices. ${ }^{13}$ However, the threshold $M$ for the transition of $T_{c}^{\max }$ from $H_{n}$ to $H_{n+1}$ is not exactly the one at which the magnetic dot generates an extra $\mathrm{V}-\mathrm{AV}$ pair. Having an equal critical temperature at two neighboring matching fields implies that the total sum of supercurrents in the superconductor is equal at both fields; this equality depends on all parameters of the system and can only be proven if calculated numerically. Therefore, although the $T_{c}^{\max }\left(H_{n}\right) \rightarrow T_{c}^{\max }\left(H_{n+1}\right)$ crossing is not exactly following the generation of an extra V-AV pair in the system, both occurrences are related, and our calculations have shown that the magnetization needed to generate the extra V-AV pair is only $\sim 10 \%$ higher than the one needed for the $T_{c}^{\max }$ transition. Therefore, in the remainder of this work, we will neglect this difference and attribute the shift of the phase boundary to the generation of an extra V-AV pair.

The results of our simulations are shown in Fig. 4 for an array of magnetic disks where the radius $R$ of the disks is varied between 300 and $700 \mathrm{~nm}$, while the period of the lattice is kept at $2 \mu \mathrm{m}$. Earlier calculations have shown that the generation of V-AV structures in superconductors under
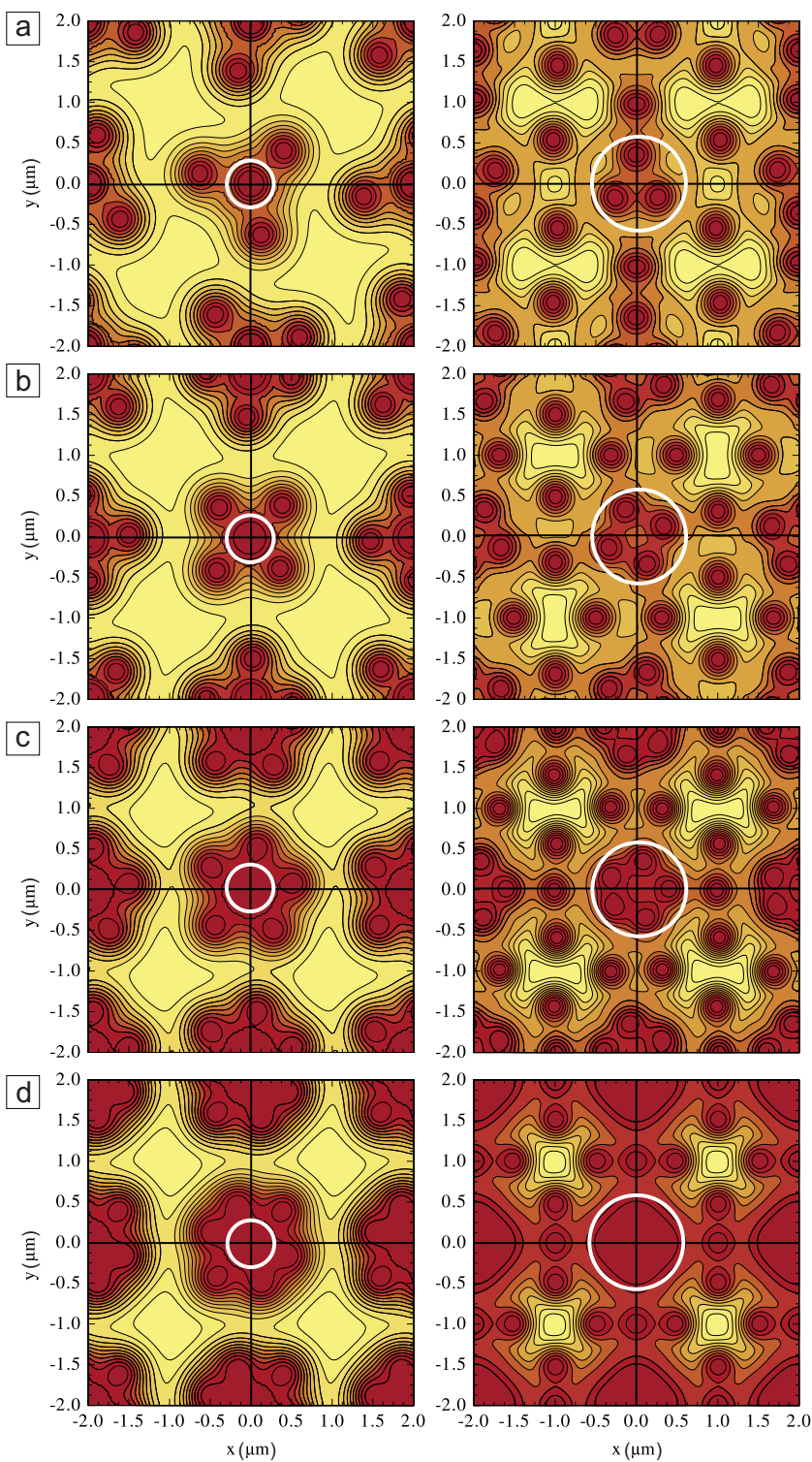

FIG. 5. (Color online) Vortex-antivortex patterns found for magnets with radii $R=300 \mathrm{~nm}$ (left panel) and $R=600 \mathrm{~nm}$ (right panel). The presented Cooper-pair density plots (low or high density-dark or light color) show vortex-antivortex configurations for (a) $N=3$, (b) $N=4$, (c) $N=5$, and (d) $N=6$. White circles represent the position of the central dot.

magnetic lattices is dependent on the lattice constant as well. ${ }^{12}$ The presented $M-R$ phase boundary in Fig. 4, delimiting regions of constant number of nucleated $\mathrm{V}-\mathrm{AV}$ pairs per magnetic dot, was calculated for parameters corresponding to the experimentally determined ones. The simulation was performed at a constant temperature $T=0.80 T_{c}$, where $T_{c}$ is the critical temperature of the plain superconducting film (thus without dots or for $M=0$ ), and in the absence of any applied uniform field $(H=0)$.

A direct comparison between the magnetization values of the experimental (Fig. 3) and theoretical (Fig. 4) $M-R$ phase boundaries reveals a good qualitative agreement. The main result is that the threshold magnetization for the nucleation of the first and subsequent vortex-antivortex pairs decreases 

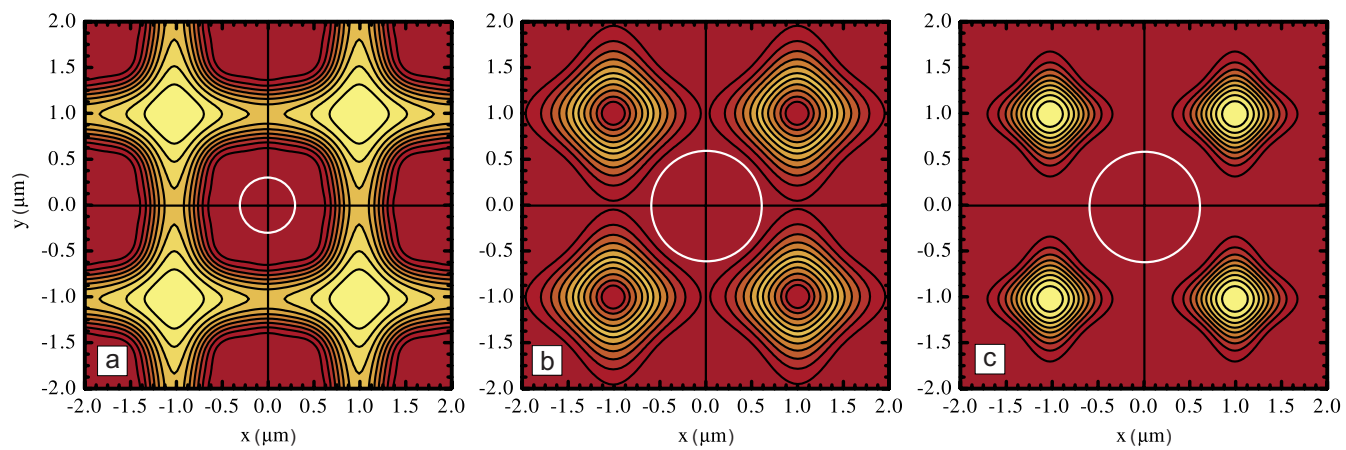

FIG. 6. (Color online) Cooper-pair density contour plots illustrating states with locally destroyed superconductivity around the magnets, with (a) $N=0$ (obtained for $R=300 \mathrm{~nm}$ ), (b) $N=1$ (obtained for $R=600 \mathrm{~nm}$ ), and (c) $N=0(R=600 \mathrm{~nm})$. White circles represent the position of the central dot.

as the dots are made larger (see Fig. 4), since the positive flux above each disk increases. This behavior perfectly corresponds to the behavior of critical $M$ for the $T_{c}^{\max }\left(H_{n}\right) \rightarrow T_{c}^{\max }\left(H_{n+1}\right)$ transition, as shown in Fig. 3. This finding indicates that the shift of the phase boundary can be further increased by increasing the size of the magnetic dots. In the theoretical phase boundary in Fig. 4, one can also see that the superconducting state is suppressed at sufficiently high magnetization of the dots. This is due to the fact that the stray field and density of induced (anti)vortices may become sufficiently large to destroy the superconducting state. In the experimental $M-R$ phase boundary, the normal state is not reached since the temperature is lowered as $M$ is increased. In between the vortex-antivortex states and the normal state, there also exists an intermediate state of localized superconductivity (discussed below).

Although the idea of generating more V-AV pairs by making bigger dots at a constant magnetization is appealing, we cannot neglect the influence of the simultaneously diminishing distance between the dots. It has been shown that reducing the period of the lattice (thus reducing the space in between the dots) can lead to the destruction of superconductivity with increasing magnetization of the dots, without any V-AV pairs appearing. ${ }^{12}$ In what follows, we will address the influence of decreasing effectively the dot interdistance. For a fixed period of the magnetic lattice, we show in Fig. 5 the contour plots of the Cooper-pair density of representative $\mathrm{V}-\mathrm{AV}$ states found with increasing magnetization for a choice of small and big dots. Each column in Fig. 5 shows the evolution of the order parameter as the magnetization is increased in order to generate three to six V-AV pairs. For the smaller dots (left panel of Fig. 5), the stray fields of the dots do not overlap and there exist regions virtually without any field at interstitial sites. This results in vortex configurations in which antivortices remain bound to the magnet. In contrast to that, larger magnetic disks share antivortices in a lattice due to the enhanced superposition of the stray fields between the disks (right panel of Fig. 5).

Neglecting the detailed structure of the Cooper-pair density and just considering the number of present V-AV pairs, Fig. 5 shows no difference between the two dot sizes. This can actually be seen better from Fig. 4 where at the considered temperature, all dot sizes generate eight $\mathrm{V}-\mathrm{AV}$ pairs
$(N=8)$ before going to the normal state. Thus, although the surface available for the AV in interstitial positions becomes smaller, no influence is seen on the maximum number of V-AV pairs. Still, for larger dot radii, more complex phenomena due to particularly small dot interspacing can be expected. We should also emphasize here the structural differences between vortex states under the magnets when $R$ increases. In the case of small magnetic disks, multiquanta vortices are located under the dots, which split into an arrangement of individual vortices under larger disks. However, we found no evidence of this last phenomenon in our experiment, most likely due to the multidomain state of the dots in the demagnetized state, which tends to suppress the superconducting state above the dots. ${ }^{14}$

As a last point, we would like to discuss the intermediate state of localized superconductivity which appears just before transiting to the normal state, as shown in the $M-R$ phase boundary in Fig. 4. The $|\psi|^{2}$ distribution of this peculiar state is shown in Fig. 6 for two different dot sizes. The localization of superconductivity is a direct consequence of the peaked field profile at the edge of the dots. Since, here, the field first exceeds $H_{c 2}$ when the magnetization rises, the Cooper pairs will be suppressed under and around the magnets. This suppression is thus localized in the vicinity of the magnets, particularly for small radii, while superconductivity survives at interstitial sites. Strictly speaking, with increasing magnetization of the disks with, e.g., $R=300 \mathrm{~nm}$, the number of vortex-antivortex pairs per disk changes as 1-2-3-4-5-6-7-8 $\rightarrow 0$, as all vortices and antivortices eventually collapse into a normal region around each magnetic disk [see Fig. 6(a)]. A similar phenomenon can be found for larger disks, although it is more difficult to achieve because of the weaker localization of the stray field. Nevertheless, once the field at the disk edges exceeds the critical field, the order parameter eventually becomes fully suppressed. However, antivortices that were shared in the lattice prior to this effect may remain at interstitial sites. Therefore, the reentrant vorticity will not be zero, as shown in Fig. 6(b), where one antivortex is trapped in each unit cell $(N=1$ state). For even higher magnetization, it becomes energetically favorable for this previously trapped antivortex to eventually collapse in the normal region as well [Fig. 6(c)]. 


\section{CONCLUSION}

We showed that the number of $\mathrm{V}-\mathrm{AV}$ pairs generated in a superconducting thin film covering an array of magnetic dots depends on both the magnetization $M$ and the radius $R$ of the magnetic dots. We further relate the existence of the vortexantivortex pairs in the superconductor to the experimentally observed shift in maximal critical temperature as a function of the applied homogeneous field $H$. This enables tuning of the shift of the $H-T$ phase boundary by adjusting either $M$ or $R$ (or both). An experimental $M-R$ phase boundary for a period of $2 \mu \mathrm{m}$ is determined, delimiting the regions where maximal $T_{c}$ is found at different matching fields. This phase boundary is in agreement with a theoretically predicted $M-R$ phase boundary for $n$ induced V-AV pairs per magnetic dot. At large dot magnetization, our theoretical modeling predicts the existence of a state with locally suppressed superconductivity, which is characteristic for spatially distant (i.e., small) dots. It is also concluded that the reduced spacing between the larger dots does not hamper nucleation of V-AV pairs for considered dot sizes (up to $R=680 \mathrm{~nm}$ ).

\section{ACKNOWLEDGMENTS}

This work was supported by the K.U. Leuven Research Fund GOA/2004/02 program, the Belgian IAP, the Fund for Scientific Research-Flanders (FWO-Vlaanderen), the Flemish Science Foundation (FWO-Vlaanderen), the Belgian Science Policy, the ESF-NES program, and the ESF-AQDJJ network. M.V.M. acknowledges support from EU Marie-Curie Intra-European program. A.V.S. is grateful for the support from the FWO-Vlaanderen. *werner.gillijns@fys.kuleuven.be

†victor.moshchalkov@fys.kuleuven.be

francois.peeters@ua.ac.be

${ }^{1}$ A. I. Buzdin, Rev. Mod. Phys. 77, 935 (2005).

${ }^{2}$ I. F. Lyuksyutov and V. L. Pokrovsky, Adv. Phys. 54, 67 (2005).

${ }^{3}$ A. I. Buzdin and A. S. Mel'nikov, Phys. Rev. B 67, 020503(R) (2003); A. Yu. Aladyshkin, A. I. Buzdin, A. A. Fraerman, A. S. Mel'nikov, D. A. Ryzhov, and A. V. Sokolov, ibid. 68, 184508 (2003).

${ }^{4}$ A. Yu. Aladyshkin and V. V. Moshchalkov, Phys. Rev. B 74, 064503 (2006).

${ }^{5}$ Z. Yang, M. Lange, A. Volodin, R. Szymczak, and V. V. Moshchalkov, Nat. Mater. 3, 793 (2004).

${ }^{6}$ W. Gillijns, A. Yu. Aladyshkin, M. Lange, M. J. Van Bael, and V. V. Moshchalkov, Phys. Rev. Lett. 95, 227003 (2005).

${ }^{7}$ Yu. I. Bespyatykh and W. Wasilevski, Phys. Solid State 43, 224 (2001).

${ }^{8}$ C. Bell, S. Turşucu, and J. Aarts, Phys. Rev. B 74, 214520 (2006).

${ }^{9}$ M. Lange, M. J. Van Bael, Y. Bruynseraede, and V. V. Moshchalkov, Phys. Rev. Lett. 90, 197006 (2003).

${ }^{10}$ M. V. Milošević and F. M. Peeters, Phys. Rev. B 68, 024509
(2003).

${ }^{11}$ D. J. Priour, Jr. and H. A. Fertig, Phys. Rev. Lett. 93, 057003 (2004).

${ }^{12}$ M. V. Milošević and F. M. Peeters, Phys. Rev. Lett. 93, 267006 (2004).

${ }^{13}$ M. V. Milošević and F. M. Peeters, Europhys. Lett. 70, 670 (2005).

${ }^{14}$ W. Gillijns, A. Yu. Aladyshkin, A. V. Silhanek, and V. V. Moshchalkov, Phys. Rev. B 76, 060503(R) (2007).

${ }^{15}$ W. Gillijns, A. V. Silhanek, and V. V. Moshchalkov, Phys. Rev. B 74, 220509(R) (2006).

${ }^{16}$ W. B. Zeper, F. J. A. M. Greidanus, P. F. Carcia, and C. R. Fincher, J. Appl. Phys. 65, 4971 (1989).

${ }^{17}$ D. J. Morgan and J. B. Ketterson, Phys. Rev. Lett. 80, 3614 (1998).

${ }^{18}$ M. Lange, M. J. Van Bael, and V. V. Moshchalkov, J. Magn. Magn. Mater. 240, 595 (2002).

${ }^{19}$ A. V. Silhanek, W. Gillijns, M. V. Milošević, A. Volodin, V. V. Moshchalkov, and F. M. Peeters, Phys. Rev. B 76, 100502(R) (2007).

${ }^{20}$ M. M. Doria, J. E. Gubernatis, and D. Rainer, Phys. Rev. B 39, 9573 (1989). 